\title{
A STUDY OF TRADE COMPLEMENTARITY BETWEEN GHINA AND THE BALTIC STATES AND ITS DEVELOPMENT STRATEGIES
}

\author{
Xiaosong Zheng ${ }^{1}$, Lijun Jia ${ }^{2 *}$, Jiawen Bao ${ }^{3}$ and Jiao Chen ${ }^{4}$ \\ 1), 2), 3), 4) Shanghai University, Shanghai, China
}

\section{Please cite this article as:}

Zheng, X., Jia, L., Bao, J. and Chen, J., 2018. A Study of Trade Complementarity between China and the Baltic States and its Development Strategies. Amfiteatru Economic, 20(49), pp. 788-803.

\section{Article History}

Received: 12 March 2018

Revised: 20 May 2018

Accepted: 26 June 2018

\begin{abstract}
The paper mainly studies trade complementarity between China and three Baltic States, namely Lithuania, Latvia and Estonia. The paper first introduces China and the three Baltic States' current trade situation. It then makes an empirical analysis on the trade complementarity between China and the three Baltic States by using models of revealed comparative advantage (RCA) and trade complementarity index (TCI) respectively, which reveals that complementarities of China to the Baltic States are mainly in the laborintensive products, while complementarities of the three Baltic States to China are in the resource-intensive products. However, the current structure of imported goods from the Baltic States to China is different from the results of complementarity analysis. In this study an expanded trade gravity model is used to analyze trade potential, which helps to develop feasible trade strategies and it shows that trade between China and the Baltic States needs to be fully exploited.
\end{abstract}

Keywords: Baltic States, China, complementarity, trade potential, trade gravity model

JEL Classification: F13, F14

\footnotetext{
* Corresponding author, Lijun Jia - jialijun@t.shu.edu.cn

This research is funded by General Project of National Planning Office of Philosophy and Social Science (2015, Project No.15BJL080). It is also funded by Major Project of National Planning Office of Philosophy and Social Science (2014, Project No. 14AGJ006).
} 


\section{Introduction}

In 2013, President Xi Jinping coined a strategic conception of the Silk Road Economic Belt and 21st-Century Maritime Silk Road, known as "One Belt One Road". The three Baltic States, namely Lithuania, Latvia and Estonia, are of a strategic location to connect the active East Asian economic area and the highly developed European economic area, which play an important role to the successful development of "One Belt One Road" project. Since then, the trade between China and the Baltic States has been more closely connected which has significant influences on mutual benefits of countries along the "One Belt One Road". For a more successful and improved trade situation, how to decide future development strategies of China and the Baltic States based on trade complementarity will be one of the key concerns. However, the studies on trade complementarity have never included complementarity between China and the Baltic States and this paper is written to fill in this gap and to emphasize the importance of research on Sino-Baltic trade complementarity as well.

The paper is divided into six sections. First, a brief introduction and the structure of the paper are presented and a literature review is followed after the introduction. Then the current trade patterns between China and the Baltic States are illustrated. The fourth section is an empirical analysis concerning Sino-Baltic trade complementarity by adopting models of RCA and TCI. The expanded trade gravity model is used in the fifth section to find out trade potential between China and the Baltic States. Lastly, the conclusion and Sino-Baltic trade development strategies are made and discussed.

\section{Literature review}

Since there have no empirical studies on trade complementarity between China and the Baltic States, the complementarity between other countries can be used as a reference for this study. Peridy (2005) concluded that trade complementarity among Pan-Arab countries, which included Egypt, Jordan, Morocco and Tunisia, was low and it limited future trade potential among those countries. Basu and Datta (2007) found that Bangladesh lacked trade complementarity to India by calculating the revealed comparative advantage index, which may lead to problems on trade balance. Zhou et al. (2007) examined the trade complementarity on agriculture trade between China and Australia, which a result of high level of complementarity and growing trend. Andreosso (2009) found obvious trade complementarity between South Korea and the European Union when using different indexes, such as RCA and TCI, to measure it. Lv and Xiang (2010) used the revealed comparative advantage analysis and Intra-Industry Index to identify trade complementarity between China and USA. According to Munemo (2011), the result of empirical research showed that trade complementarity of China to Southern African countries was much higher than Southern African countries to China. Hatab et al. (2012) suggested that trade complementarity of China to Egypt was increasing, while that of Egypt to China was decreasing from the analysis. Natos et al. (2014) concluded that the calculation results of the indexes, like the trade complementarity index, indicated that complementarity of Western Balkans to other EU member states was not quite favorable. Vahalik (2014) analyzed the trade complementarity among EU, China and the Association of Southeast Asian Nations (ASEAN), which implied that EU was a bigger trading partner to ASEAN than to China. Khadan and Hosein (2015) argued that the trade complementarity level of 
Caribbean Community bloc to Canada was relative low and had a decreasing trend. Kumar and Ahmed (2015) investigated India-Sir Lanka intra-industry trade and its trade complementarity through calculating a number of indexes.

All these trade complementarity studies have some implications for Sino-Baltic trade complementarity study. In the following sections a detailed study of Sino-Baltic trade complementarity is presented.

\section{China's trade with the Baltic States: the current pattern}

In this section, trade data from 2002 to 2015 of China with Lithuania, Latvia and Estonia, which are compiled from the United Nations Commodity Trade Statistics Database (UN Comtrade Database), are used to analyze their current trade pattern. In addition, the Standard Industrial Trade Classification Revision 3 (SITC Rev3) is used for trade classification, in which SITC0 to SITC4 are resource-intensive products, while SITC5 and SITC7 are capital-intensive products and SITC6 and SITC8 are labor-intensive products.

\subsection{Import and export structure between China and Lithuania}

From the databases, we find that SITC6, SITC7 and SITC8 account for the major proportions, which are $88.93 \%$ total in average from 2002 to 2015, of all the goods exported to Lithuania. Meanwhile, SITC0, SITC2 and SITC5 account for $10.88 \%$ of the total export. However, the proportion of the rest four categories is only $0.19 \%$ in average, which is negligible. That is, capital-intensive products and labor-intensive products are major parts of goods exported from China to Lithuania from 2002 to 2015, especially SITC7. Meanwhile, resource-intensive products are just a minority part of the goods exported to Lithuania.

In addition, it shows that there have been great changes in the import structure of China from Lithuania from 2002 to 2015. Overall, the import structure of China from Lithuania currently has a trend of labor-intensive products, especially SITC8, instead of the resourceintensive products.

\subsection{Import and export structure between China and Latvia}

SITC6, SITC7 and SITC8 are the majority of goods exported from China to Latvia, and the proportion of SITC6 and SITC7 has an upward trend, while SITC8 presents a downward trend, which means labor-intensive goods account for the most part of export products, following by capital-intensive products.

In addition, for the import, one of the most obvious changes happened in SITC2, rising from $18.06 \%$ to $60 \%$, while the import of SITC5 and SITC7 dropped from $19.10 \%$ and $38.04 \%$ to $5.19 \%$ and $6.62 \%$ respectively. Meanwhile, the proportion of SITC6 and SITC8 ascended as well. In conclusion, the import structure tends to be the resource-intensive goods, especially SITC2 and SITC3, instead of capital-intensive products. 


\subsection{Import and export structure between China and Estonia}

SITC7 occupies the majority of export goods from China to Estonia, but it has a dropping trend from $71.43 \%$ in 2002 to $50.32 \%$ in 2015 . On the contrary, the proportion of SITC8 and SITC6 grows steadily. The rest categories only make up a small proportion of goods exported from China to Estonia. The above data show that capital-intensive products and labor-intensive products are mainly export commodities, especially SITC7. However, the capital-intensive goods have a dropping trend, while the labor-intensive goods show a positive trend. As for the import, the import structure of China from Estonia currently has a tendency of changing to capital-intensive goods and labor-intensives goods, particularly capital-intensive products, instead of resource-intensive goods.

\section{Trade Complementarity Analysis between China and the Baltic States}

\subsection{Methodology and data source}

\subsubsection{Comparative Advantage Theory}

Smith (1776) proposed the theory of absolute advantage, which means that if the labor productivity of Country A in one product is higher than Country B, Country A will export this product. However, in reality, developed countries have absolute advantage in almost all products and still trade with other countries. Under this circumstance, Ricardo (1817) put forward the law of comparative advantage. In his opinion, even if Country A has absolute advantages in both products and Country B has absolute disadvantages, the possibility of trade would still exist if the absolute advantages are different. The comparative advantages can be determined by factor endowments, differences in labor productivity or production levels and technology characteristics.

In this research, revealed comparative advantage (RCA) index is used to measure the comparative advantage of China and the Baltic States. The notion of RCA was proposed by Balassa (1965), which was defined as the ratio between the proportion of a particular commodity in the total export of the country and the share of this commodity in the world total export. RCA can be written as:

$$
\mathrm{RCA}_{\mathrm{i}}^{\mathrm{k}}=\left(\mathrm{X}_{\mathrm{i}}^{\mathrm{k}} / \mathrm{X}_{\mathrm{i}}\right) /\left(\mathrm{X}_{\mathrm{w}}^{\mathrm{k}} / \mathrm{X}_{\mathrm{w}}\right)
$$

Where:

$$
\begin{array}{ll}
\mathrm{RCA}_{\mathrm{i}}^{\mathrm{k}} & - \text { comparative advantage of export product } \mathrm{k} \text { in country } \mathrm{i} \\
\mathrm{X}_{\mathrm{i}}^{\mathrm{k}} & - \text { total export of product } \mathrm{k} \text { in country } \mathrm{i} \\
\mathrm{X}_{\mathrm{W}}^{\mathrm{k}} & - \text { total export of product } \mathrm{k} \text { in the whole world } \mathrm{w} \\
\mathrm{X}_{\mathrm{i}} & - \text { total export of country } \mathrm{i} \\
\mathrm{X}_{\mathrm{W}} & - \text { total export of all countries }
\end{array}
$$

This index can be regarded as an indicator for evaluating comparative advantage and international competitiveness. In general, if RCA $<0.8$, it means that this commodity owns little world competitiveness; if RCA is between 0.8 and 1.25 , it starts to enjoy certain comparative advantage; if RCA is between 1.25 and 2.5 , the competitiveness is considerable; if RCA $>2.5$, this kind of goods is strongly competitive. 


\subsubsection{Trade Complementarity Index}

One of the most common methods to measure trade complementarity and the trade relationship between different countries is using trade complementarity index (TCI). Drysdale (1969) defined TCI as product of the revealed comparative advantage index measured by the export of one country in a certain commodity and the revealed comparative disadvantage index measured by the import of the other country in that commodity. TCI can be expressed as:

$$
\begin{aligned}
& \operatorname{TCI}_{1 \mathrm{ij}}^{\mathrm{k}}=\operatorname{RCA}_{\mathrm{Ni}}^{\mathrm{k}} \times \mathrm{RCA}_{\mathrm{Nj}}^{\mathrm{k}} \\
& \operatorname{RCA}_{\mathrm{Nj}}^{\mathrm{k}}=\left(\mathrm{M}_{\mathrm{j}}^{\mathrm{k}} / \mathrm{M}_{\mathrm{j}}\right) /\left(\mathrm{M}_{\mathrm{w}}^{\mathrm{k}} / \mathrm{M}_{\mathrm{w}}\right)
\end{aligned}
$$

Where:

$$
\begin{array}{ll}
\mathrm{TCI}_{i j}^{\mathrm{k}} & - \text { trade complementarity index of country } i \text { and } \mathrm{j} \text { in product } \mathrm{k} \\
\mathrm{RCA}_{\mathrm{Ki}}^{\mathrm{k}} & - \text { comparative advantage of export product } \mathrm{k} \text { in country } \mathrm{i} \\
\mathrm{RCA}_{\mathrm{NIj}}^{\mathrm{k}} & - \text { comparative advantage of export product } \mathrm{k} \text { in country } \mathrm{j} \\
\mathrm{M}_{\mathrm{j}}^{\mathrm{k}} & - \text { total import of product } \mathrm{k} \text { in country } \mathrm{j} \\
\mathrm{M}_{\mathrm{i}} & - \text { total import of country } \mathrm{j} \\
\mathrm{M}_{\mathrm{w}}^{\mathrm{k}} & \text { - total import of product } \mathrm{k} \text { in the world } \mathrm{w} \\
\mathrm{M}_{\mathrm{w}} & \text { - total import of the world } \mathrm{w}
\end{array}
$$

Generally, if TCI $>1$, the trade complementarity is strong, otherwise it is weak. When the export commodity of one country is identical with the import commodity of the other country, TCI tends to be greater.

In the following analysis, the trade data between China and Lithuania, Latvia and Estonia are selected from UN Comtrade. Meanwhile, all the data are based on SITC Rev3.

\subsection{Empirical analysis}

\subsubsection{RCA analysis}

The RCA for China and the Baltic States can be seen in table no. 1, table no. 2, table no. 3 and table no. 4 below. From these tables, it can be seen that the comparative advantages of China mainly occur in the labor-intensive goods from 2002 to 2015 , especially SITC8. Meanwhile, the SITC7 has achieved certain competitiveness as well. Considering the three Baltic States, the comparative advantages occurred in the resource-intensive goods, particularly SITC0, SITC1 and SITC2. These results are consistent with the resource endowment in China and the Baltic States.

Table no. 1: RCA of China from 2002 to 2015

\begin{tabular}{lllllllllll}
\hline Year & SITC0 & SITC1 & SITC2 & SITC3 & SITC4 & SITC5 & SITC6 & SITC7 & SITC8 & SITC9 \\
\hline $\mathbf{2 0 0 2}$ & 0.796 & 0.318 & 0.461 & 0.288 & 0.079 & 0.457 & 1.183 & 0.965 & 2.482 & 0.048 \\
$\mathbf{2 0 0 3}$ & 0.715 & 0.251 & 0.383 & 0.268 & 0.064 & 0.423 & 1.154 & 1.080 & 2.327 & 0.050 \\
$\mathbf{2 0 0 4}$ & 0.604 & 0.240 & 0.315 & 0.238 & 0.061 & 0.418 & 1.206 & 1.153 & 2.226 & 0.043 \\
$\mathbf{2 0 0 5}$ & 0.575 & 0.194 & 0.308 & 0.188 & 0.095 & 0.442 & 1.217 & 1.207 & 2.204 & 0.055
\end{tabular}


Economic Interferences $7 \varepsilon$

\begin{tabular}{lllllllllll}
\hline Year & SITC0 & SITC1 & SITC2 & SITC3 & SITC4 & SITC5 & SITC6 & SITC7 & SITC8 & SITC9 \\
\hline $\mathbf{2 0 0 6}$ & 0.547 & 0.162 & 0.243 & 0.132 & 0.105 & 0.447 & 1.281 & 1.253 & 2.220 & 0.063 \\
$\mathbf{2 0 0 7}$ & 0.498 & 0.148 & 0.212 & 0.131 & 0.058 & 0.468 & 1.252 & 1.280 & 2.214 & 0.043 \\
$\mathbf{2 0 0 8}$ & 0.435 & 0.144 & 0.225 & 0.135 & 0.075 & 0.531 & 1.334 & 1.369 & 2.255 & 0.027 \\
$\mathbf{2 0 0 9}$ & 0.442 & 0.156 & 0.198 & 0.126 & 0.054 & 0.451 & 1.221 & 1.439 & 2.141 & 0.024 \\
$\mathbf{2 0 1 0}$ & 0.463 & 0.159 & 0.183 & 0.113 & 0.047 & 0.504 & 1.227 & 1.450 & 2.189 & 0.018 \\
$\mathbf{2 0 1 1}$ & 0.469 & 0.162 & 0.184 & 0.099 & 0.049 & 0.564 & 1.302 & 1.471 & 2.284 & 0.024 \\
$\mathbf{2 0 1 2}$ & 0.444 & 0.163 & 0.174 & 0.089 & 0.048 & 0.525 & 1.323 & 1.441 & 2.389 & 0.013 \\
$\mathbf{2 0 1 3}$ & 0.427 & 0.149 & 0.168 & 0.092 & 0.054 & 0.515 & 1.349 & 1.439 & 2.373 & 0.014 \\
$\mathbf{2 0 1 4}$ & 0.411 & 0.154 & 0.181 & 0.097 & 0.056 & 0.536 & 1.380 & 1.350 & 2.261 & 0.020 \\
$\mathbf{2 0 1 5}$ & 0.405 & 0.174 & 0.177 & 0.120 & 0.058 & 0.511 & 1.368 & 1.276 & 2.044 & 0.015 \\
\hline
\end{tabular}

Source: UN Comtrade database and own calculations

Table no. 2: RCA of Lithuania from 2002 to 2015

\begin{tabular}{lllllllllll}
\hline Year & SITC0 & SITC1 & SITC2 & SITC3 & SITC4 & SITC5 & SITC6 & SITC7 & SITC8 & SITC9 \\
\hline $\mathbf{2 0 0 2}$ & 1.656 & 0.774 & 2.199 & 2.072 & 0.509 & 0.718 & 0.894 & 0.642 & 1.501 & 0.056 \\
$\mathbf{2 0 0 3}$ & 1.853 & 0.649 & 2.052 & 2.054 & 0.372 & 0.714 & 0.763 & 0.665 & 1.514 & 0.052 \\
$\mathbf{2 0 0 4}$ & 1.917 & 0.827 & 1.884 & 2.441 & 0.419 & 0.747 & 0.764 & 0.551 & 1.471 & 0.083 \\
$\mathbf{2 0 0 5}$ & 2.047 & 1.529 & 1.592 & 2.164 & 0.445 & 0.794 & 0.750 & 0.562 & 1.317 & 0.207 \\
$\mathbf{2 0 0 6}$ & 2.407 & 2.144 & 1.359 & 1.694 & 0.596 & 0.882 & 0.768 & 0.595 & 1.398 & 0.189 \\
$\mathbf{2 0 0 7}$ & 2.767 & 2.412 & 1.533 & 1.021 & 0.612 & 1.265 & 0.814 & 0.621 & 1.459 & 0.238 \\
$\mathbf{2 0 0 8}$ & 2.520 & 1.826 & 1.109 & 1.508 & 0.683 & 1.300 & 0.695 & 0.543 & 1.285 & 0.245 \\
$\mathbf{2 0 0 9}$ & 2.593 & 2.169 & 1.088 & 1.585 & 0.654 & 1.177 & 0.788 & 0.491 & 1.283 & 0.299 \\
$\mathbf{2 0 1 0}$ & 2.546 & 2.638 & 1.092 & 1.559 & 0.328 & 1.169 & 0.776 & 0.519 & 1.247 & 0.302 \\
$\mathbf{2 0 1 1}$ & 2.276 & 2.998 & 1.011 & 1.480 & 0.294 & 1.263 & 0.745 & 0.545 & 1.201 & 0.315 \\
$\mathbf{2 0 1 2}$ & 2.496 & 2.844 & 1.080 & 1.449 & 0.356 & 1.228 & 0.776 & 0.531 & 1.159 & 0.348 \\
$\mathbf{2 0 1 3}$ & 2.497 & 3.340 & 1.090 & 1.379 & 0.449 & 1.152 & 0.810 & 0.537 & 1.247 & 0.313 \\
$\mathbf{2 0 1 4}$ & 2.345 & 3.586 & 1.148 & 1.156 & 0.615 & 1.221 & 0.827 & 0.580 & 1.293 & 0.505 \\
$\mathbf{2 0 1 5}$ & 2.294 & 3.140 & 1.395 & 1.603 & 0.811 & 1.321 & 0.847 & 0.495 & 1.255 & 0.367 \\
\hline
\end{tabular}

Source: UN Comtrade database and own calculations

Table no. 3: RCA of Latvia from 2002 to 2015

\begin{tabular}{ccccccccccc}
\hline Year & SITC0 & SITC1 & SITC2 & SITC3 & SITC4 & SITC5 & SITC6 & SITC7 & SITC8 & SITC9 \\
\hline $\mathbf{2 0 0 2}$ & 1.210 & 3.115 & 9.125 & 0.164 & 0.093 & 0.575 & 2.138 & 0.205 & 1.443 & 0.068 \\
$\mathbf{2 0 0 3}$ & 1.205 & 2.066 & 9.447 & 0.146 & 0.152 & 0.581 & 2.093 & 0.230 & 1.415 & 0.071 \\
$\mathbf{2 0 0 4}$ & 1.238 & 2.683 & 7.156 & 0.450 & 0.313 & 0.565 & 2.103 & 0.259 & 1.368 & 0.502 \\
$\mathbf{2 0 0 5}$ & 1.591 & 2.931 & 6.168 & 0.723 & 0.420 & 0.567 & 1.851 & 0.323 & 1.112 & 0.985 \\
$\mathbf{2 0 0 6}$ & 1.895 & 3.302 & 5.525 & 0.373 & 0.535 & 0.724 & 1.832 & 0.399 & 1.128 & 0.995 \\
$\mathbf{2 0 0 7}$ & 1.743 & 4.936 & 5.185 & 0.280 & 0.342 & 0.766 & 1.778 & 0.463 & 0.967 & 0.897 \\
$\mathbf{2 0 0 8}$ & 2.070 & 4.992 & 4.085 & 0.209 & 0.352 & 0.874 & 1.845 & 0.553 & 0.949 & 0.905
\end{tabular}




\begin{tabular}{lllllllllll}
\hline Year & SITC0 & SITC1 & SITC2 & SITC3 & SITC4 & SITC5 & SITC6 & SITC7 & SITC8 & SITC9 \\
\hline $\mathbf{2 0 0 9}$ & 2.091 & 3.856 & 3.990 & 0.376 & 0.271 & 0.790 & 1.679 & 0.598 & 0.870 & 0.726 \\
$\mathbf{2 0 1 0}$ & 2.105 & 4.899 & 4.173 & 0.354 & 0.537 & 0.752 & 1.736 & 0.539 & 0.880 & 0.660 \\
$\mathbf{2 0 1 1}$ & 1.773 & 5.217 & 3.615 & 0.483 & 0.456 & 0.757 & 1.678 & 0.570 & 0.826 & 1.004 \\
$\mathbf{2 0 1 2}$ & 2.210 & 6.109 & 3.311 & 0.463 & 0.445 & 0.671 & 1.741 & 0.549 & 0.777 & 1.132 \\
$\mathbf{2 0 1 3}$ & 2.130 & 6.404 & 3.348 & 0.461 & 0.584 & 0.681 & 1.550 & 0.568 & 0.845 & 1.279 \\
$\mathbf{2 0 1 4}$ & 1.965 & 6.496 & 3.613 & 0.489 & 0.589 & 0.694 & 1.425 & 0.609 & 0.809 & 1.291 \\
$\mathbf{2 0 1 5}$ & 1.988 & 4.812 & 3.903 & 0.609 & 0.417 & 0.706 & 1.451 & 0.640 & 0.785 & 0.717 \\
\hline
\end{tabular}

Source: UN Comtrade database and own calculations

Table no.4: RCA of Estonia from 2002 to 2015

\begin{tabular}{lllllllllll}
\hline Year & SITC0 & SITC1 & SITC2 & SITC3 & SITC4 & SITC5 & SITC6 & SITC7 & SITC8 & SITC9 \\
\hline $\mathbf{2 0 0 2}$ & 1.829 & 0.928 & 3.523 & 0.592 & 1.258 & 0.544 & 1.557 & 0.681 & 1.449 & 0.000 \\
$\mathbf{2 0 0 3}$ & 1.619 & 1.233 & 3.480 & 0.448 & 0.795 & 0.592 & 1.521 & 0.744 & 1.479 & 0.001 \\
$\mathbf{2 0 0 4}$ & 1.460 & 1.522 & 3.011 & 0.446 & 0.698 & 0.500 & 1.299 & 0.825 & 1.469 & 0.787 \\
$\mathbf{2 0 0 5}$ & 1.253 & 1.508 & 2.676 & 0.589 & 0.691 & 0.477 & 1.207 & 0.872 & 1.293 & 1.613 \\
$\mathbf{2 0 0 6}$ & 1.161 & 1.966 & 2.384 & 1.120 & 0.771 & 0.474 & 1.099 & 0.799 & 1.224 & 1.366 \\
$\mathbf{2 0 0 7}$ & 1.269 & 3.168 & 2.397 & 0.951 & 0.687 & 0.514 & 1.224 & 0.743 & 1.312 & 1.187 \\
$\mathbf{2 0 0 8}$ & 1.242 & 2.701 & 2.241 & 0.718 & 0.777 & 0.586 & 1.336 & 0.814 & 1.390 & 0.983 \\
$\mathbf{2 0 0 9}$ & 1.290 & 1.765 & 1.833 & 1.224 & 0.747 & 0.512 & 1.210 & 0.788 & 1.260 & 0.824 \\
$\mathbf{2 0 1 0}$ & 1.363 & 1.946 & 1.982 & 1.052 & 0.860 & 0.490 & 1.176 & 0.811 & 1.334 & 0.756 \\
$\mathbf{2 0 1 1}$ & 1.211 & 1.861 & 1.575 & 0.981 & 0.472 & 0.519 & 1.104 & 0.937 & 1.289 & 0.827 \\
$\mathbf{2 0 1 2}$ & 1.252 & 2.041 & 1.569 & 0.954 & 0.486 & 0.541 & 1.195 & 0.919 & 1.222 & 0.858 \\
$\mathbf{2 0 1 3}$ & 1.342 & 2.069 & 1.728 & 0.612 & 0.652 & 0.584 & 1.261 & 0.977 & 1.314 & 0.902 \\
$\mathbf{2 0 1 4}$ & 1.335 & 1.959 & 1.909 & 0.729 & 0.632 & 0.496 & 1.118 & 0.955 & 1.240 & 1.155 \\
$\mathbf{2 0 1 5}$ & 1.334 & 1.480 & 2.081 & 1.079 & 0.627 & 0.466 & 1.087 & 0.862 & 1.207 & 1.081 \\
\hline
\end{tabular}

Source: UN Comtrade database and own calculations

\subsubsection{TCI analysis}

Table no. 5 to table no. 10 demonstrates the TCI between China and the Baltic States respectively. The specific analysis is discussed in the following parts.

- TCI between China and Lithuania

From table no. 5 it is known that China does not have obvious trade complementarity with Lithuania in resource-intensive goods from 2002 to 2015, since all the TCI are less than 1. For the capital-intensive goods, China lacks complementarity with Lithuania generally as well. However, the complementarity of China to Lithuania in the labor-intensive goods is quite strong and steady. As a result, China should strengthen the trade in labor-intensive goods and limit the trade in resource-intensive goods. 
Table no. 5: TCI of Sino-Lithuania from 2002 to 2015

\begin{tabular}{lllllllllll}
\hline Year & SITC0 & SITC1 & SITC2 & SITC3 & SITC4 & SITC5 & SITC6 & SITC7 & SITC8 & SITC9 \\
\hline $\mathbf{2 0 0 2}$ & 0.856 & 0.306 & 0.565 & 0.504 & 0.124 & 0.489 & 1.538 & 0.822 & 1.374 & 0.027 \\
$\mathbf{2 0 0 3}$ & 0.809 & 0.227 & 0.429 & 0.439 & 0.090 & 0.447 & 1.440 & 0.958 & 1.356 & 0.022 \\
$\mathbf{2 0 0 4}$ & 0.731 & 0.259 & 0.321 & 0.397 & 0.092 & 0.447 & 1.537 & 0.966 & 1.345 & 0.013 \\
$\mathbf{2 0 0 5}$ & 0.718 & 0.246 & 0.290 & 0.331 & 0.115 & 0.447 & 1.389 & 0.965 & 1.283 & 0.028 \\
$\mathbf{2 0 0 6}$ & 0.775 & 0.262 & 0.206 & 0.203 & 0.129 & 0.486 & 1.431 & 1.067 & 1.427 & 0.013 \\
$\mathbf{2 0 0 7}$ & 0.729 & 0.247 & 0.167 & 0.151 & 0.061 & 0.546 & 1.433 & 1.202 & 1.550 & 0.014 \\
$\mathbf{2 0 0 8}$ & 0.694 & 0.259 & 0.196 & 0.215 & 0.086 & 0.561 & 1.227 & 1.072 & 1.509 & 0.011 \\
$\mathbf{2 0 0 9}$ & 0.788 & 0.359 & 0.175 & 0.245 & 0.073 & 0.560 & 1.178 & 0.803 & 1.416 & 0.011 \\
$\mathbf{2 0 1 0}$ & 0.817 & 0.361 & 0.132 & 0.236 & 0.047 & 0.590 & 1.093 & 0.821 & 1.248 & 0.010 \\
$\mathbf{2 0 1 1}$ & 0.752 & 0.378 & 0.127 & 0.187 & 0.041 & 0.631 & 1.138 & 0.935 & 1.287 & 0.014 \\
$\mathbf{2 0 1 2}$ & 0.768 & 0.421 & 0.123 & 0.166 & 0.050 & 0.599 & 1.196 & 0.847 & 1.274 & 0.008 \\
$\mathbf{2 0 1 3}$ & 0.773 & 0.417 & 0.124 & 0.157 & 0.065 & 0.577 & 1.287 & 0.910 & 1.444 & 0.008 \\
$\mathbf{2 0 1 4}$ & 0.691 & 0.436 & 0.137 & 0.145 & 0.070 & 0.627 & 1.390 & 0.930 & 1.571 & 0.016 \\
$\mathbf{2 0 1 5}$ & 0.660 & 0.444 & 0.170 & 0.216 & 0.073 & 0.649 & 1.409 & 0.830 & 1.410 & 0.012 \\
\hline
\end{tabular}

Source: UN Comtrade database and own calculations

From table no. 6 it can be observed that Lithuania has quite obvious trade complementarity in some categories of the resource-intensive goods from 2002 to 2015, which are SITC2, SITC3 and SITC4, especially for SITC2. However, Lithuania does not have trade complementarity to China in SITC0 and SITC1. Concerning the capital-intensive goods, Lithuania has some fading complementarities. For SITC7, the complementarity has lost since 2008. Meanwhile, for the labor-intensive goods, Lithuania does not have complementarity with China in most years from 2002 to 2015, especially for SITC8. Overall, the trade complementarity of Lithuania to China mainly reflects the resourceintensive goods, particularly SITC2, and SITC5 of the capital-intensive goods. Consequently, Lithuania should export more resource-intensive goods and chemical products and less labor-intensive products.

Table no. 6: TCI of Lithuania-Sino from 2002 to 2015

\begin{tabular}{ccccccccccc}
\hline Year & SITC0 & SITC1 & SITC2 & SITC3 & SITC4 & SITC5 & SITC6 & SITC7 & SITC8 & SITC9 \\
\hline $\mathbf{2 0 0 2}$ & 0.327 & 0.131 & 2.865 & 1.214 & 2.333 & 1.322 & 1.573 & 1.006 & 0.286 & 0.082 \\
$\mathbf{2 0 0 3}$ & 0.283 & 0.116 & 2.759 & 1.132 & 2.467 & 1.151 & 1.441 & 1.073 & 0.367 & 0.037 \\
$\mathbf{2 0 0 4}$ & 0.368 & 0.122 & 2.788 & 1.276 & 2.757 & 1.151 & 1.226 & 0.988 & 0.447 & 0.023 \\
$\mathbf{2 0 0 5}$ & 0.345 & 0.183 & 2.798 & 1.248 & 1.670 & 1.095 & 1.036 & 0.945 & 0.458 & 0.051 \\
$\mathbf{2 0 0 6}$ & 0.363 & 0.277 & 2.407 & 1.181 & 1.661 & 1.130 & 0.898 & 1.050 & 0.516 & 0.013 \\
$\mathbf{2 0 0 7}$ & 0.343 & 0.314 & 2.432 & 0.901 & 1.980 & 1.212 & 0.879 & 1.128 & 0.578 & 0.022 \\
$\mathbf{2 0 0 8}$ & 0.374 & 0.420 & 3.064 & 1.366 & 2.047 & 1.037 & 0.650 & 0.915 & 0.557 & 0.038 \\
$\mathbf{2 0 0 9}$ & 0.427 & 0.507 & 3.227 & 1.676 & 1.988 & 1.168 & 0.841 & 0.666 & 0.487 & 0.031 \\
$\mathbf{2 0 1 0}$ & 0.485 & 0.517 & 2.449 & 1.854 & 1.242 & 1.095 & 0.664 & 0.648 & 0.429 & 0.179 \\
$\mathbf{2 0 1 1}$ & 0.464 & 0.654 & 2.330 & 1.691 & 0.914 & 1.025 & 0.598 & 0.708 & 0.403 & 0.415
\end{tabular}




\begin{tabular}{lllllllllll}
\hline Year & SITC0 & SITC1 & SITC2 & SITC3 & SITC4 & SITC5 & SITC6 & SITC7 & SITC8 & SITC9 \\
\hline $\mathbf{2 0 1 2}$ & 0.599 & 0.815 & 2.342 & 1.744 & 1.343 & 1.018 & 0.617 & 0.643 & 0.391 & 0.535 \\
$\mathbf{2 0 1 3}$ & 0.669 & 0.830 & 2.458 & 1.587 & 1.302 & 0.982 & 0.622 & 0.693 & 0.418 & 0.649 \\
$\mathbf{2 0 1 4}$ & 0.670 & 0.862 & 2.431 & 1.489 & 1.187 & 1.018 & 0.735 & 0.746 & 0.457 & 0.874 \\
$\mathbf{2 0 1 5}$ & 0.788 & 1.064 & 3.044 & 1.883 & 1.186 & 1.100 & 0.684 & 0.718 & 0.465 & 0.808 \\
\hline
\end{tabular}

Source: UN Comtrade database and own calculations

- TCI between China and Latvia

Table no. 7 shows that China does not have trade complementarity in resource-intensive goods with Latvia from 2002 to 2015. Meanwhile, this situation is getting worse. Considering the capital-intensive commodities, China does not have complementarity with Latvia during most time of the period. For SITC7, it has certain complementarity in 2015, but fluctuation occurs as well. As for the labor-intensive products, China owns steady trade complementarity with Latvia from 2002 to 2015. In conclusion, China should partly export the labor-intensive products to Latvia

Table no.7: TCI of Sino-Latvia from 2002 to 2015

\begin{tabular}{ccccccccccc}
\hline Year & SITC0 & SITC1 & SITC2 & SITC3 & SITC4 & SITC5 & SITC6 & SITC7 & SITC8 & SITC9 \\
\hline 2002 & 1.303 & 0.834 & 0.464 & 0.284 & 0.148 & 0.542 & 1.662 & 0.754 & 2.328 & 0.000 \\
2003 & 1.096 & 0.577 & 0.455 & 0.246 & 0.098 & 0.479 & 1.692 & 0.867 & 2.191 & 0.000 \\
2004 & 0.900 & 0.586 & 0.422 & 0.252 & 0.084 & 0.432 & 1.796 & 0.852 & 1.911 & 0.028 \\
2005 & 0.895 & 0.509 & 0.326 & 0.207 & 0.112 & 0.413 & 1.626 & 0.930 & 1.899 & 0.065 \\
2006 & 0.820 & 0.521 & 0.216 & 0.115 & 0.120 & 0.430 & 1.647 & 1.090 & 2.153 & 0.058 \\
2007 & 0.716 & 0.480 & 0.215 & 0.100 & 0.049 & 0.423 & 1.540 & 1.192 & 2.110 & 0.044 \\
2008 & 0.773 & 0.463 & 0.193 & 0.114 & 0.075 & 0.566 & 1.618 & 1.134 & 2.182 & 0.027 \\
2009 & 0.940 & 0.428 & 0.145 & 0.145 & 0.071 & 0.496 & 1.390 & 0.856 & 1.822 & 0.038 \\
2010 & 0.967 & 0.411 & 0.150 & 0.109 & 0.059 & 0.579 & 1.516 & 0.920 & 1.825 & 0.034 \\
2011 & 0.854 & 0.374 & 0.127 & 0.094 & 0.068 & 0.559 & 1.654 & 1.086 & 1.881 & 0.047 \\
2012 & 0.818 & 0.404 & 0.187 & 0.083 & 0.078 & 0.485 & 1.521 & 1.060 & 2.034 & 0.024 \\
2013 & 0.792 & 0.389 & 0.127 & 0.083 & 0.079 & 0.494 & 1.608 & 0.996 & 2.145 & 0.030 \\
2014 & 0.715 & 0.548 & 0.155 & 0.083 & 0.069 & 0.523 & 1.653 & 0.994 & 2.060 & 0.041 \\
2015 & 0.663 & 0.629 & 0.166 & 0.122 & 0.061 & 0.510 & 1.637 & 1.010 & 1.740 & 0.019 \\
\hline
\end{tabular}

Source: UN Comtrade database and own calculations

Table no. 8 clearly shows that Latvia has extremely strong trade complementarity in SITC2 of the resource-intensive goods with China from 2002 to 2015, followed by SITC1. Although the other categories of the resource-intensive goods present improvements, they still lack complementarity with China. For the capital-intensive goods, Latvia does not own trade complementarity with China. For the labor-intensive commodities, the complementarity of Latvia to China has a downward trend. Considering SITC5, Latvia used to have complementarity with China from 2002 to 2015, but it lost complementarity in 2015. Considering SITC7, it is lack of complementarity on the whole. As a result, Latvia should focus on exporting resource-intensive commodities, especially SITC2. 
Table no. 8: TCI of Latvia-Sino from 2002 to 2015

\begin{tabular}{lllllllllll}
\hline Year & SITC0 & SITC1 & SITC2 & SITC3 & SITC4 & SITC5 & SITC6 & SITC7 & SITC8 & SITC9 \\
\hline 2002 & 0.368 & 0.425 & 21.314 & 0.114 & 0.138 & 0.710 & 2.587 & 0.243 & 0.746 & 0.010 \\
2003 & 0.302 & 0.264 & 23.292 & 0.101 & 0.267 & 0.632 & 2.417 & 0.278 & 0.890 & 0.006 \\
2004 & 0.376 & 0.303 & 19.591 & 0.343 & 0.573 & 0.607 & 2.023 & 0.305 & 1.011 & 0.037 \\
2005 & 0.439 & 0.422 & 18.341 & 0.511 & 0.577 & 0.614 & 1.682 & 0.382 & 0.875 & 0.099 \\
2006 & 0.486 & 0.567 & 15.665 & 0.287 & 0.722 & 0.752 & 1.474 & 0.492 & 0.905 & 0.072 \\
2007 & 0.408 & 0.924 & 16.014 & 0.219 & 0.641 & 0.796 & 1.365 & 0.556 & 0.798 & 0.061 \\
2008 & 0.486 & 1.161 & 14.357 & 0.179 & 0.623 & 0.858 & 1.304 & 0.646 & 0.791 & 0.087 \\
2009 & 0.501 & 0.850 & 14.638 & 0.325 & 0.400 & 0.742 & 1.464 & 0.715 & 0.640 & 0.050 \\
2010 & 0.578 & 1.112 & 14.118 & 0.314 & 0.670 & 0.703 & 1.296 & 0.618 & 0.662 & 0.211 \\
2011 & 0.513 & 1.460 & 12.174 & 0.435 & 0.506 & 0.694 & 1.148 & 0.635 & 0.591 & 0.724 \\
2012 & 0.766 & 1.936 & 10.976 & 0.437 & 0.577 & 0.599 & 1.189 & 0.600 & 0.570 & 0.944 \\
2013 & 0.788 & 1.903 & 11.198 & 0.426 & 0.640 & 0.597 & 1.010 & 0.622 & 0.580 & 1.447 \\
2014 & 0.784 & 1.983 & 11.650 & 0.486 & 0.559 & 0.604 & 1.039 & 0.659 & 0.532 & 1.403 \\
2015 & 0.961 & 2.004 & 12.422 & 0.640 & 0.392 & 0.611 & 0.963 & 0.707 & 0.529 & 0.737 \\
\hline
\end{tabular}

Source: UN Comtrade database and own calculations

- TCI between China and Estonia

Table no. 9 reveals that China does not have any trade complementarities in resourceintensive commodities to Estonia, especially for SITC4. Among the capital-intensive products, China lacks complementarity in SITC5 but has it in SITC7 to Estonia. For the labor-intensive goods, China has palpable complementarity to Estonia. However, the complementarity gradually decreases from 2002 to 2015. In general, the trade complementarity of China to Estonia mainly appears in the labor-intensive goods and machinery and transport equipment, which China should export more in the future.

Table no. 9: TCI of Sino-Estonia from 2002 to 2015

\begin{tabular}{ccccccccccc}
\hline Year & SITC0 & SITC1 & SITC2 & SITC3 & SITC4 & SITC5 & SITC6 & SITC7 & SITC8 & SITC9 \\
\hline $\mathbf{2 0 0 2}$ & 1.272 & 0.553 & 0.557 & 0.220 & 0.089 & 0.423 & 1.728 & 0.935 & 1.841 & 0.000 \\
$\mathbf{2 0 0 3}$ & 1.144 & 0.449 & 0.437 & 0.156 & 0.035 & 0.371 & 1.664 & 1.147 & 1.629 & 0.004 \\
$\mathbf{2 0 0 4}$ & 0.864 & 0.449 & 0.365 & 0.157 & 0.037 & 0.342 & 1.775 & 1.120 & 1.622 & 0.046 \\
$\mathbf{2 0 0 5}$ & 0.766 & 0.374 & 0.325 & 0.140 & 0.051 & 0.356 & 1.563 & 1.229 & 1.544 & 0.094 \\
$\mathbf{2 0 0 6}$ & 0.658 & 0.269 & 0.208 & 0.162 & 0.053 & 0.347 & 1.472 & 1.166 & 1.510 & 0.106 \\
$\mathbf{2 0 0 7}$ & 0.587 & 0.457 & 0.181 & 0.135 & 0.032 & 0.378 & 1.556 & 1.150 & 1.757 & 0.071 \\
$\mathbf{2 0 0 8}$ & 0.577 & 0.464 & 0.133 & 0.124 & 0.074 & 0.498 & 1.762 & 1.221 & 2.106 & 0.029 \\
$\mathbf{2 0 0 9}$ & 0.689 & 0.413 & 0.128 & 0.167 & 0.054 & 0.418 & 1.445 & 1.044 & 1.944 & 0.028 \\
$\mathbf{2 0 1 0}$ & 0.721 & 0.472 & 0.116 & 0.123 & 0.018 & 0.456 & 1.583 & 1.199 & 1.971 & 0.020 \\
$\mathbf{2 0 1 1}$ & 0.609 & 0.470 & 0.093 & 0.101 & 0.019 & 0.463 & 1.553 & 1.471 & 1.713 & 0.034 \\
$\mathbf{2 0 1 2}$ & 0.523 & 0.467 & 0.110 & 0.086 & 0.020 & 0.461 & 1.528 & 1.445 & 1.778 & 0.019 \\
$\mathbf{2 0 1 3}$ & 0.563 & 0.435 & 0.113 & 0.073 & 0.026 & 0.434 & 1.606 & 1.464 & 1.908 & 0.022
\end{tabular}




\begin{tabular}{ccccccccccc}
\hline Year & SITC0 & SITC1 & SITC2 & SITC3 & SITC4 & SITC5 & SITC6 & SITC7 & SITC8 & SITC9 \\
\hline $\mathbf{2 0 1 4}$ & 0.521 & 0.443 & 0.125 & 0.093 & 0.021 & 0.442 & 1.550 & 1.259 & 1.868 & 0.040 \\
$\mathbf{2 0 1 5}$ & 0.530 & 0.434 & 0.138 & 0.141 & 0.019 & 0.423 & 1.527 & 1.129 & 1.587 & 0.027 \\
\hline
\end{tabular}

Source: UN Comtrade database and own calculations

Table no. 10 demonstrates that in the resource-intensive goods, Estonia has trade complementarity only in SITC2 and the complementarity is significant. For SITC3, it may be a future star for the complementarity of Estonia to China, since it has an increasing trend from 2002 to 2015. However, for the other categories in the resource-intensive goods, it lacks complementarity. In addition, Estonia does not own obvious complementarities from 2002 to 2015 to China in almost all categories of the capital-intensive goods and laborintensive goods. Among those products, SITC7 has a steady and optimistic trend. And hence, Estonia should export SITC2 and SITC3, particularly SITC2, which it has trade complementarity, to China.

Table no. 10: TCI of Estonia-Sino from 2002 to 2015

\begin{tabular}{lllllllllll}
\hline Year & SITC0 & SITC1 & SITC2 & SITC3 & SITC4 & SITC5 & SITC6 & SITC7 & SITC8 & SITC9 \\
\hline $\mathbf{2 0 0 2}$ & 0.556 & 0.127 & 8.230 & 0.411 & 1.865 & 0.672 & 1.884 & 0.805 & 0.750 & 0.000 \\
$\mathbf{2 0 0 3}$ & 0.405 & 0.158 & 8.580 & 0.309 & 1.392 & 0.644 & 1.756 & 0.901 & 0.930 & 0.000 \\
$\mathbf{2 0 0 4}$ & 0.444 & 0.172 & 8.243 & 0.340 & 1.279 & 0.537 & 1.250 & 0.973 & 1.086 & 0.058 \\
$\mathbf{2 0 0 5}$ & 0.346 & 0.217 & 7.957 & 0.416 & 0.949 & 0.517 & 1.096 & 1.031 & 1.018 & 0.162 \\
$\mathbf{2 0 0 6}$ & 0.298 & 0.338 & 6.760 & 0.863 & 1.041 & 0.493 & 0.884 & 0.984 & 0.982 & 0.098 \\
$\mathbf{2 0 0 7}$ & 0.297 & 0.593 & 7.403 & 0.743 & 1.285 & 0.535 & 0.939 & 0.892 & 1.083 & 0.081 \\
$\mathbf{2 0 0 8}$ & 0.291 & 0.628 & 7.877 & 0.616 & 1.373 & 0.576 & 0.944 & 0.951 & 1.158 & 0.094 \\
$\mathbf{2 0 0 9}$ & 0.309 & 0.389 & 6.723 & 1.058 & 1.104 & 0.482 & 1.055 & 0.941 & 0.927 & 0.057 \\
$\mathbf{2 0 1 0}$ & 0.374 & 0.441 & 6.705 & 0.931 & 1.072 & 0.458 & 0.878 & 0.929 & 1.004 & 0.242 \\
$\mathbf{2 0 1 1}$ & 0.351 & 0.521 & 5.306 & 0.884 & 0.523 & 0.476 & 0.755 & 1.044 & 0.923 & 0.597 \\
$\mathbf{2 0 1 2}$ & 0.434 & 0.647 & 5.201 & 0.899 & 0.631 & 0.483 & 0.816 & 1.005 & 0.895 & 0.715 \\
$\mathbf{2 0 1 3}$ & 0.496 & 0.615 & 5.780 & 0.567 & 0.714 & 0.512 & 0.822 & 1.070 & 0.902 & 1.020 \\
$\mathbf{2 0 1 4}$ & 0.532 & 0.598 & 6.154 & 0.726 & 0.600 & 0.431 & 0.816 & 1.034 & 0.815 & 1.256 \\
$\mathbf{2 0 1 5}$ & 0.645 & 0.616 & 6.623 & 1.134 & 0.589 & 0.404 & 0.722 & 0.951 & 0.814 & 1.111 \\
\hline
\end{tabular}

Source: UN Comtrade database and own calculations

\section{Trade Potential Analysis between China and the Baltic States}

\subsection{Model construction and data sources}

- Trade Gravity Model between China and the three Baltic States

Tinbergen (1962) and Poyhonen (1963) brought the gravity model into the trade field for the first time and formed the trade gravity model. They thought the bilateral trade between two countries was in proportion to their economic aggregates and was inversely proportional to their distance. In recent years, many researchers have used the trade gravity model to evaluate the factors that affect the bilateral trade and measure the trade 
potential though comparing the estimated results and the actual values, such as the research done by Swapan and Biswanath (2007) and Zhang et al. (2010).

Based on the previous research and the purpose of this research, we constructs an expanded trade gravity model between China and the Baltic States by adding new explanatory variables into the traditional one. The new equation is as follows:

$$
\begin{aligned}
\operatorname{lnXijt}= & \alpha_{0}+\alpha_{1} \operatorname{InGDPper}_{\mathrm{it}}+\alpha_{2} \operatorname{InGDPper}_{\mathrm{jt}}+\alpha_{\mathrm{a}} \text { disr }_{\mathrm{ij}}+\alpha_{4} \operatorname{Inpopu}_{\mathrm{jt}}+\alpha_{5} \text { euzone }_{\mathrm{jt}}+ \\
& \alpha_{6} \mathrm{WTO}_{\mathrm{ijt}}+\mu_{\mathrm{ij}}
\end{aligned}
$$

Where:

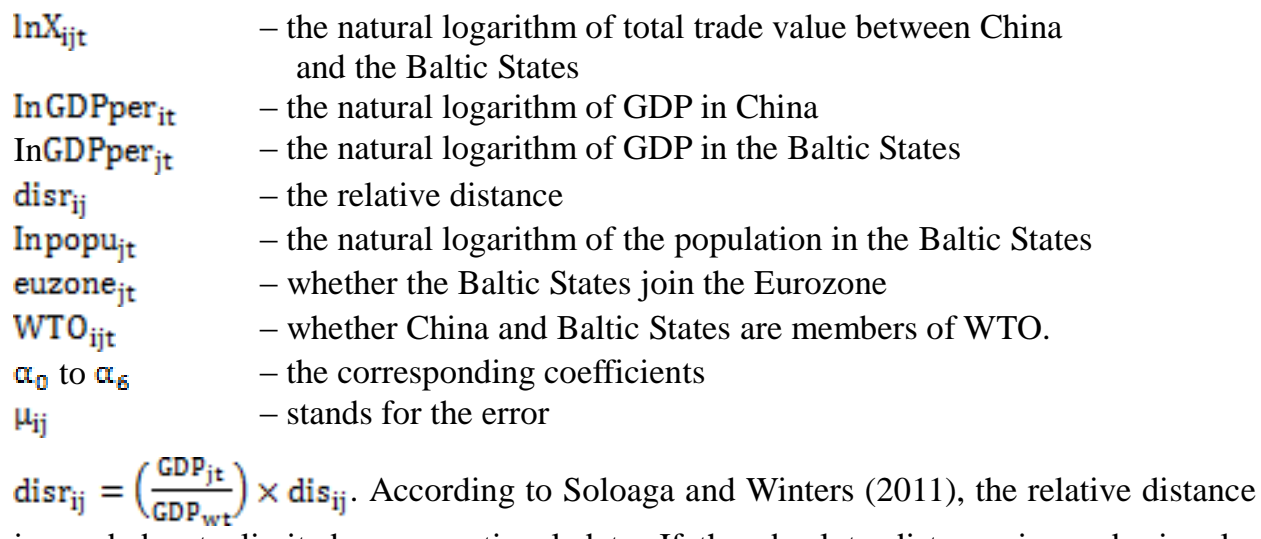
is used due to limited cross-sectional data. If the absolute distance is used, singular matrix will occur, which will cause the correct function of the estimation.

- Data sources

The new trade gravity model is based on panel data, the time span of which is from 1996 to 2015 and the cross sections of which are Lithuania, Latvia and Estonia. Among those, the trade data between China and the Baltic States are selected from the UN Comtrade Database. The data of GDP per capita and population are from World Development Indicators of the World Bank, so is the population data. The distance variable is the distance between Beijing and Vilnius, Riga and Tallinn, which is obtained from CEPII database.

\subsection{Empirical results and analysis}

There are three regression estimation methods for panel data, namely pooled regression, random effect model and fixed effect model. Since the Hausman test accepts random effect model, we used it and got the following results:

$$
\begin{aligned}
& \ln X \mathrm{ijt}=53.889+0.654 \operatorname{InGDPper}_{\mathrm{it}}+1.277 \mathrm{InGDPper}_{\mathrm{jt}}-6.992 \mathrm{disr}_{\mathrm{ij}}+ \\
& +0.662 \mathrm{Inpopu}_{\mathrm{jt}}-0.199 \text { euzone }_{\mathrm{jt}}+0.905 \mathrm{WTO}_{\mathrm{ijt}}
\end{aligned}
$$

From the equation 5, it is known that the greatest factor that affects trade value between China and the Baltic States is distance. As the distance increases $1 \%$, the total trade value decreases by $6.992 \%$, which means distance is the main factor hindering the trade 
development. As a result, it is necessary to construct a good transportation infrastructure, such as rail, air and maritime transport, in order to weaken this strong negative impact. In terms of GDP per capita of the Baltic States, if it rises by $1 \%$, the total trade will increase by $1.277 \%$. However, if the GDP per capita of China increases by $1 \%$, the trade will only rise by $0.654 \%$. This shows that the trade structure is quite unreasonable. As for the population factor, the trade value will rise by $0.662 \%$ for every increased $1 \%$ of it. Whether the Baltic States join Eurozone and whether China and the Baltic States belong to WTO have the same effect on total trade value as expected.

\subsection{Trade potential and the possible development countermeasures}

The research gets the analog value T' of the trade value between China and the Baltic States from 1996 to 2015 by substituting the parameters into Equation 5. After that, it is easy to estimate the trade potential by dividing the actual trade value by the analog one, which has been shown in Table 11. If the result is less than 0.8, huge potential exists there. If the result is between 0.8 and 1.2, it is in a pioneering state of potential. If the result is over 1.2, the potential is limited and reform will be needed.

From Table no. 11, it is found that the trade potential values between China and Lithuania, Latvia, and Estonia are all around 1 and fluctuation occurs rarely from 1996 to 2015. Meanwhile, China and the Baltic States are in a pioneering state of potential. However, the results show that the trade potential between China and the Baltic States has not been exploited properly and the trade structure has not been optimized as well.

Based on the liner regression equation, this research proposes the following possible development countermeasures:

- China and the three Baltic States can construct a wide range of transport infrastructure, such as rail transport, maritime transport and air transport, to reduce trade costs, in order to completely exploit trade potential and promote future trade development.

- China and Lithuania, Latvia, and Estonia should reform their trade structures based on their own trade complementarities. China should strengthen the production and export of labor-intensive products, while the Baltic States should pay more attention to the export of resource-intensive goods, especially crude materials, so as to fully use the trade potential between China and the Baltic States.

Table no. 11: Trade potential value from 2002 to 2015

\begin{tabular}{cccc}
\hline Year & Lithuania & Latvia & Estonia \\
\hline $\mathbf{1 9 9 6}$ & 0.98 & 0.99 & 0.96 \\
$\mathbf{1 9 9 7}$ & 0.98 & 0.94 & 0.96 \\
$\mathbf{1 9 9 8}$ & 0.99 & 1.00 & 0.97 \\
$\mathbf{1 9 9 9}$ & 1.00 & 1.00 & 0.95 \\
$\mathbf{2 0 0 0}$ & 1.01 & 1.01 & 1.05 \\
$\mathbf{2 0 0 1}$ & 1.03 & 1.03 & 1.12 \\
$\mathbf{2 0 0 2}$ & 1.00 & 0.99 & 1.03 \\
\hline
\end{tabular}




\begin{tabular}{cccc}
\hline Economic Interferences & & & \\
\hline Year & Lithuania & Latvia & Estonia \\
\hline $\mathbf{2 0 0 3}$ & 1.00 & 1.00 & 1.00 \\
$\mathbf{2 0 0 4}$ & 1.01 & 1.00 & 1.00 \\
$\mathbf{2 0 0 5}$ & 1.01 & 1.01 & 1.01 \\
$\mathbf{2 0 0 6}$ & 1.01 & 1.01 & 1.02 \\
$\mathbf{2 0 0 7}$ & 1.00 & 1.00 & 0.99 \\
$\mathbf{2 0 0 8}$ & 1.00 & 0.99 & 0.98 \\
$\mathbf{2 0 0 9}$ & 0.99 & 0.98 & 0.97 \\
$\mathbf{2 0 1 0}$ & 1.00 & 1.00 & 1.00 \\
$\mathbf{2 0 1 1}$ & 1.00 & 1.01 & 1.00 \\
$\mathbf{2 0 1 2}$ & 1.01 & 1.01 & 1.01 \\
$\mathbf{2 0 1 3}$ & 1.00 & 1.00 & 1.00 \\
$\mathbf{2 0 1 4}$ & 0.99 & 1.01 & 0.99 \\
$\mathbf{2 0 1 5}$ & 1.00 & 1.00 & 1.00 \\
\hline
\end{tabular}

\section{Conclusion}

With the proposed concept of "One Belt One Road" project in China, the trade between China and the Baltic States has been highly promoted. However, to the best of our knowledge scholars have never studied trade complementarity between China and the Baltic States, namely Lithuania, Latvia and Estonia. This paper is written to fill in the gap and to emphasize the importance of research on Sino-Baltic trade complementarity as well.

In this research we made a detailed analysis of trade complementarity between China and the Baltic States by calculating the RCA and TCI indexes. Meanwhile, trade potential and possible development strategies are measured through the establishment of an expanded trade gravity model. Based on the research we can draw the following conclusions and development strategies.

First, trade complementarity is mainly reflected on factor endowments and comparative advantages, which means that China has trade complementarity with the Baltic States in the labor-intensive commodities and Lithuania, Latvia and Estonia have it mainly in the resource-intensive goods, especially the crude materials. However, the current import and export structure of China has certain difference with the trade complementarity results, mainly reflecting in the import structure of China from the Baltic States. Currently, the Baltic States have a tendency of exporting the capital-intensive goods and labor-intensive goods, particularly in Lithuania and Estonia. Consequently, for the future trade development, the trade structure needs to follow the trade complementarity results, which means that China should export more labor-intensive goods and import more resource-intensive goods, especially the crude materials.

Secondly, after constructing the expanded trade gravity model, we found that distance is the greatest factor that influences total trade value between China and the Baltic States, followed by the GDP factor. Meanwhile, by comparing the analog value from the regression of the trade gravity model and the actual value of China and the Baltic States, 
it is found that the results are roughly the same between China and Lithuania, Latvia and Estonia from 1996 to 2015, which are around 1. That is, the exploitation of the trade potential between China and the Baltic States in recent years is not satisfied. One of the methods to exploit the trade potential and promote the future trade development between China and the Baltic States is to strengthen the transport infrastructure construction to weaken the negative influence brought by distance.

\section{References}

Andreosso, B., 2009. Economic structural complementarity: how viable is the Korea-EU FTA?. Journal of Economic Studies, 36(2), pp. 147-167.

Basu, S. and Datta, D., 2007. India-Bangladesh Trade Deficit and Misaligned Bilateral Exchange Rate: Can Bangladesh Draw Lessons from Indonesia?. Journal of the Asia Pacific Economy, 12(1), pp. 76-102.

Balassa, B., 1965. Trade Liberalization and 'Revealed' Comparative Advantage. Manchester School of Economics and Social Studies, 33, pp. 99-123.

Drysdale, P., 1969. Japan, Australia and New Zealand: the Prospects for Western Integration. Economic Record, 45(3), pp. 321-342.

Hatab, A.R., Shoumann, N.A. and Huo, X., 2012. Exploring Egypt- China bilateral trade: dynamics and prospects. Journal of Economic Studies, 39(3), pp. 314-326.

Khadan, J. and Hosein, R., 2015. Trade, Economic and Welfare Impacts of the CARICOM-Canada Free Trade Agreement. Social and Economic Studies, 64, pp. 103-150.

Kumar, S. and Ahmed, S., 2015. Intra-Industry Trade and Trade Complementarity: Evidence from India-Sri Lanka Bilateral Trade. Journal of International Economics, 6(2), pp. 38-70.

Lv, J. and Xiang, L., 2010. Empirical Analysis of Bilateral Trade Complementarity between China and the U.S.A.. In: IEEE Wuhan Section, International Conference on Management and Service Science. Wuhan, China, 24-26 August 2010. Piscataway, N.J.: IEEE.

Munemo, J., 2011. Trade between China and South Africa: Prospects of a Successful SACU- China Free Trade Agreement. African Development Review, 25(3), pp. 303329.

Natos, D., Staboulis, C. and Tsakiridou, E., 2014. Agricultural Trade Integration in Western Balkans: Orientation and Complementarity. Agricultural Economics Review, 15(2), pp. 85-99.

Peridy, N., 2005. Toward a PAN-Arab Free Trade Area: Assessing Trade Potential Effects of the Agadir Agreement. Developing Economies, 43(3), pp. 329-345.

Poyhonen, P., 1963. A Tentative Model for the Volume of Trade between Countries. Weltwirtschaftliches Archive, 90(1), pp. 93-100.

Ricardo, D., 1817. On the principles of political economy and taxation. London: Electric Book Corporation.

Soloage, I. and Winters, L., 2001. Regionalism in the Nineties: What Effect on Trade? North American Journal of Economics and Finance, 12, pp. 1-29. 
Smith, A., 1776. An inquiry into the nature and causes of the wealth of nations. Oxford: Clarendon Press.

Swapan, B. and Biswanath, B., 2007. Gains and Losses of India-China Trade Cooperation - A Gravity Model Impact Analysis. St. Louis: Federal Reserve Bank of St Louis.

Tinbergen, J., 1962. Shaping the World Economy: Suggestions for an International Economic Policy. New York: Twentieth Century Fund.

Vahalik, B., 2014. Regional Bilateral Trade Analysis of the European Union, China and ASEAN. In: Faculty Of Business And Economics, Mendel University, Brno, Czech Republic, Enterprise and the Competitive Environment 2014 conference. Brno, Czech Republic, 6-7 March 2014.

Zhou, Z., Wu, Y. and Si, W., 2007. Evolving Patterns of Agricultural Trade between Australia and China. Australasian Agribusiness Review, 15, pp. 27-45.

Zhang, H., Xie, J. and Zheng, J., 2010. Determinants and Potential of China-Africa Agricultural Trade: An Empirical Study Based on Gravity Model. In: IEEE., International Conference on Management Science and Engineering. Melbourne, Australia, 24-26 November 2010. Melbourne: IEEE. 